\title{
Origins of spatial, temporal and numerical cognition: Insights from comparative psychology
}

\author{
Daniel B.M. Haun ${ }^{1,2,3}$, Fiona M. Jordan ${ }^{2}$, Giorgio Vallortigara ${ }^{4}$ and Nicky S. Clayton ${ }^{5}$
}

\author{
${ }^{1}$ Max Planck Institute for Evolutionary Anthropology, Deutscher Platz 6, 04103, Leipzig, Germany \\ ${ }^{2}$ Max Planck Institute for Psycholinguistics, Wundtlaan 1, 6525 XD Nijmegen, The Netherlands \\ ${ }^{3}$ Department of Psychology, University of Portsmouth, King Henry I Street, Portsmouth PO1 2DY \\ ${ }^{4}$ Centre for Mind/Brain Sciences, University of Trento, Corso Bettini, 31, I-38068 Rovereto, Italy \\ ${ }^{5}$ Department of Experimental Psychology, University of Cambridge, Downing Street, Cambridge CB2 3EB, UK
}

\begin{abstract}
Contemporary comparative cognition has a large repertoire of animal models and methods, with concurrent theoretical advances that are providing initial answers to crucial questions about human cognition. What cognitive traits are uniquely human? What are the speciestypical inherited predispositions of the human mind? What is the human mind capable of without certain types of specific experiences with the surrounding environment? Here, we review recent findings from the domains of space, time and number cognition. These findings are produced using different comparative methodologies relying on different animal species, namely birds and non-human great apes. The study of these species not only reveals the range of cognitive abilities across vertebrates, but also increases our understanding of human cognition in crucial ways.
\end{abstract}

Researching human cognition through the study of other species

'He who understands baboon would do more towards Metaphysics than Locke'

(Charles Darwin, 1838, Notebook M84e)

In this short note, 21 years before publication of the Origin of Species, Charles Darwin recognised the value of studying animal cognition for human psychology. Implicit here is the idea that cognitive processes are biological adaptations with evolutionary histories and, therefore, cognition is tractable to between-species mapping of similarities and differences in cognitive abilities. The past two decades have seen an increase in the number of species studied and the types of methodological approaches used in the growing field of comparative cognition [1,2]. Concurrently, this work has become interdisciplinary between biology, psychology, neuroscience, genetics, linguistics and anthropology. Here, we review lines of evidence in which the study of other animal species has informed current understanding of the structure and evolution of three core domains of human cognition: space, number and time. We demonstrate

Corresponding author: Haun, D.B.M. (haun@eva.mpg.de). how different methodologies in comparative cognition not only reveal the range of cognitive abilities within the animal kingdom, but also increase understanding of human cognition in crucial ways, enabling researchers to address seemingly intractable questions such as: (i) are some cognitive capacities in place at birth? (ii) what is the evolutionary endowment of human cognition? And (iii) which cognitive abilities are uniquely human?

\section{Are some cognitive capacities in place at birth?}

Rigorous controlled-rearing experiments with non-human animals enabled scientists to establish what mechanisms are present at birth and the impact of specific experiences on shaping basic perceptual-motor capacities [3]. For example, 'visual cliff' studies showed that the ability to judge depth through motion parallax is in place at birth in a variety of animal species [4]. This pioneering work, however, did not venture into more complex cognitive capacities, such as the cognition of space, number or time. Recently, however, it has been proposed that complex human cognitive achievements, such as mathematics

\footnotetext{
Glossary

Altricial: species in which the young are relatively immobile after birth or hatching and must be cared for by adults.

Convergent evolution: a process where similar characteristics evolve in unrelated groups of organisms, also called analogy.

Heritability (narrow sense): degree to which individual phenotypes are determined by the additive effects of genes transmitted from the parents: mathematically it is expressed as the ratio of the additive genetic variance to the total phenotypic variance.

Homology/homologous evolution: similar characteristics that are shared by groups of organisms owing to descent from a common ancestor.

Model organism: species that are extensively studied with the expectation that conclusions drawn are relevant to other organisms.

Phylogeny: the evolutionary history of a group of organisms or populations, usually described by a tree structure showing the hierarchy of relatedness between groups

Phylogenetics: the modern field of evolutionary biology; uses a broad range of computational methods to construct trees and networks of how groups of organisms are related and how their characteristics evolve.

Precocial: species in which the young are relatively mature and mobile soon after birth or hatching.

Taxa: a named population sharing similar characteristics; for example, a species (singular: taxon)
} 
and geometry, which are uniquely human in their full linguistic and symbolic realisation, rest nevertheless on a set of core knowledge systems that humans share with other animals [5]. Because of their limited behavioural repertoire, the study of cognitive capacities in human infants is limited, as it is in the young of altricial species (see Glossary) in general. Therefore, investigating species that are precocial with regard to their pattern of motor and sensory development makes possible sophisticated behavioural analyses of early ages, enabling us to investigate the influence of specific experiences on inborn cognition.

\section{Precocial animal models}

Being a precocial species, the domestic chick (Gallus gallus) has been a successful animal model system for tackling some classic issues in developmental psychology, such as the origins of both social (e.g. biological motion [6] and causal agency [7]) and physical cognition (e.g. object permanence [8]). The heuristic value of research with chicks for human developmental studies has been particularly apparent in the area of early social predispositions. At their first exposure to point-light animation sequences, visually inexperienced chicks exhibit a spontaneous preference to approach biological motion patterns [9]. These findings stimulated a substantial body of research concerning perception of biological motion in human newborns (e.g. $[10,11])$ that revealed astonishingly similar predispositions.

Chicks have also been used recently to investigate the origins of space and number cognition. Neurobiological evidence suggests basic homology in the avian and mammalian brain for a key neural structure involved in space cognition (hippocampal formation) and possibly for associative areas involved in number cognition (mesopallium) [12].

For example, much interest has been devoted to the issue of how humans and animals regain their sense of direction when they become disoriented. There appears to be impressive sensitivity to surface layout geometry in guiding spatial reorientation $[13,14]$. For example, when an animal observes the hiding of a target in one corner of a rectangular enclosure, and is then inertially disoriented, it subsequently shows selective searching at the two geometrically correct corners of the enclosure, avoiding the corners with incorrect metric (short/long) and sense (left/ right) properties in the arrangement of surfaces [13,14].

Competing theories have been formulated as to how animals and humans reorient themselves in these circumstamces; for example, Fodorian modular encapsulated computations of the shape of the extended surfaces layout [15]; combination of environmental cues weighted according to their experienced reliability [16]; and image-matching processes operating on panoramic $2 \mathrm{D}$ projections of current and remembered environments [17]. Several empirical studies have been carried out in both vertebrates [18-20] and invertebrates [21] in attempt to determine the relative merits of the different theories. One approach has been to investigate whether the system for reorientation does have some of the hallmarks of a Fodorian module, such as specific genetic bases [22] and specific neural mechanisms $[23,24]$, and whether it develops in the absence of relevant experience of navigating in a geometrically structured layout. The last issue is important even irrespective of a Fodorian approach, and can be successfully addressed using controlled-rearing studies.

In rectangular enclosures, geometric information is fully available because of the presence of metrically distinct surfaces connected at right angles and two principal axes of symmetry. In circular enclosures, by contrast, this geometric information is removed and there is an infinite number of principal axes. In C-shaped enclosures, neither right angles nor differences in wall length are available, although the first principal axis is still usable to encode shape. Chicks reared soon after hatching in home-cages with these different geometric shapes proved to be equally capable of learning and performing navigational tasks based on geometric information $[18,25]$. This suggests that effective use of geometric information for spatial reorientation in principle does not require experience in environments with right angles and metrically distinct surfaces. Recently, further evidence that at least some aspects of spatial representations are present at birth arose from single-cell recording studies, showing that, when rat pups explore an open environment outside the nest for the first time, head-direction cells show adult-like properties from the beginning; place and grid cells are also present from the beginning but their selectivity refines gradually [26,27].

It could be argued that the pattern of development of precocial species might be peculiar, and not generalisable to humans (however, rats are also an altricial species). Nonetheless, these findings provide evidence that, in principle, a capacity can develop fully in the absence of a specific experiential contribution. Some differences between altricial and precocial species might turn out to be the byproduct of maturation of other mechanisms rather than the outcome of specific learning. For instance, the ability to complete partly occluded objects mentally (amodal completion) is apparent in chicks soon after hatching [28], which could be taken as evidence for mechanisms that do not require experience; in human infants, this ability is only present from approximately four months of age [29]. Recently, however, it has been shown that when stroboscopic motion is used instead of continuous motion (the former being processed early in development by subcortical structures) human neonates only a few hours old show evidence of amodal completion similar to that of chicks [30]. Thus, in altricial species, maturation of other brain areas seems to be necessary to exhibit in behaviour the mental competences that are predisposed at birth. Similarly, the results obtained in chicks [31] suggest that basic features of natural geometry are largely in place at birth; however, in humans, language and other types of nongeometric experience might influence the development of uniquely human forms of spatial knowledge [32].

Although specific experiences might not be crucial in encoding surface geometry, it could be that they are important in the combined use of geometric and non-geometric information (e.g. features such as the colour of a wall) for reorientation. Some results with a less precocial species of fish (Archocentrus nigrofasciatus) suggest that, when geometric and non-geometric information are set in conflict, rearing experience could affect the relative dominance 
of featural (landmark) and geometric information [33]. The same effect was not observed in chicks [18], suggesting that experiences have different roles in the relative reliance of use of geometric and non-geometric information in altricial and precocial species; however, this will require confirmation through more species comparisons.

Numerical cognition in chicks is also apparent early in development and parallels closely that observed in human infants. For example, in small identical object arrays, infants represent the total continuous extent of the visual array rather than its numerosity [34] or, according to some authors, both continuous extent and numerosity [35]. However, objects with contrasting sets of properties provoke infants to respond to the number of objects rather than to their continuous extent [36]. Similarly, newborn chicks have been tested for their sensitivity to number versus continuous extent of artificial objects that they had been reared with soon after hatching [37]. When the objects were similar, chicks chose the set of objects of larger numerosity, irrespective of the number of objects that they had been reared with. However, when chicks were reared with objects that differed in their aspect (colour, size and shape) and then tested with completely novel objects (but controlled for continuous extent), they chose to associate with a set of objects comprising the same number of elements that they had been reared with during imprinting. Early availability of small numerosity discrimination by chicks suggests that these abilities are in place at birth [38]. Even basic arithmetic seems available in very young chicks [39], which are capable of computing exact addition and subtraction on small numbers of social partners, with no previous experience of appearance and disappearance of such objects (Figure 1). Finally, a disposition to map the numerical number line from left to right, possibly as a result of left visual hemifield (right hemisphere) dominance [40], has been reported [37]. Hence, the disposition to map number and space is apparent very early in development in these precocial species.

\section{What is the evolutionary endowment of human cognition?}

Inherited cognitive capacities and preferences are not necessarily present at birth, but might emerge only later in ontogeny. Children might be inherently prepared to acquire an ability or preference over time [41]. We here refer to the question of whether any variance found in a late-blooming human cognitive capacity is due to speciestypical genetic variance [42]. For example, capacities for relational thought [43], false belief reasoning [44] and the ability to think about the past and imagine the future [45] do not fully develop before roughly four years of age. Although these sophisticated capacities are not present at birth, there is no a priori reason to exclude the possibility that heritable factors construct childrens' abilities in these late-blooming cognitive domains. Given that they develop later in life, data neither from human infants nor precocial species will shed light on the nature of these inherited predispositions.

\section{Closely related animal models}

Taxonomically informed cross-species comparisons within our immediate primate family, the great apes, offer a way to investigate the evolutionary history of late-blooming human cognitive skills. For this purpose, 'heritable' cognitive characteristics should be seen as part of the evolutionary endowment of the species, that is, inherited from a last

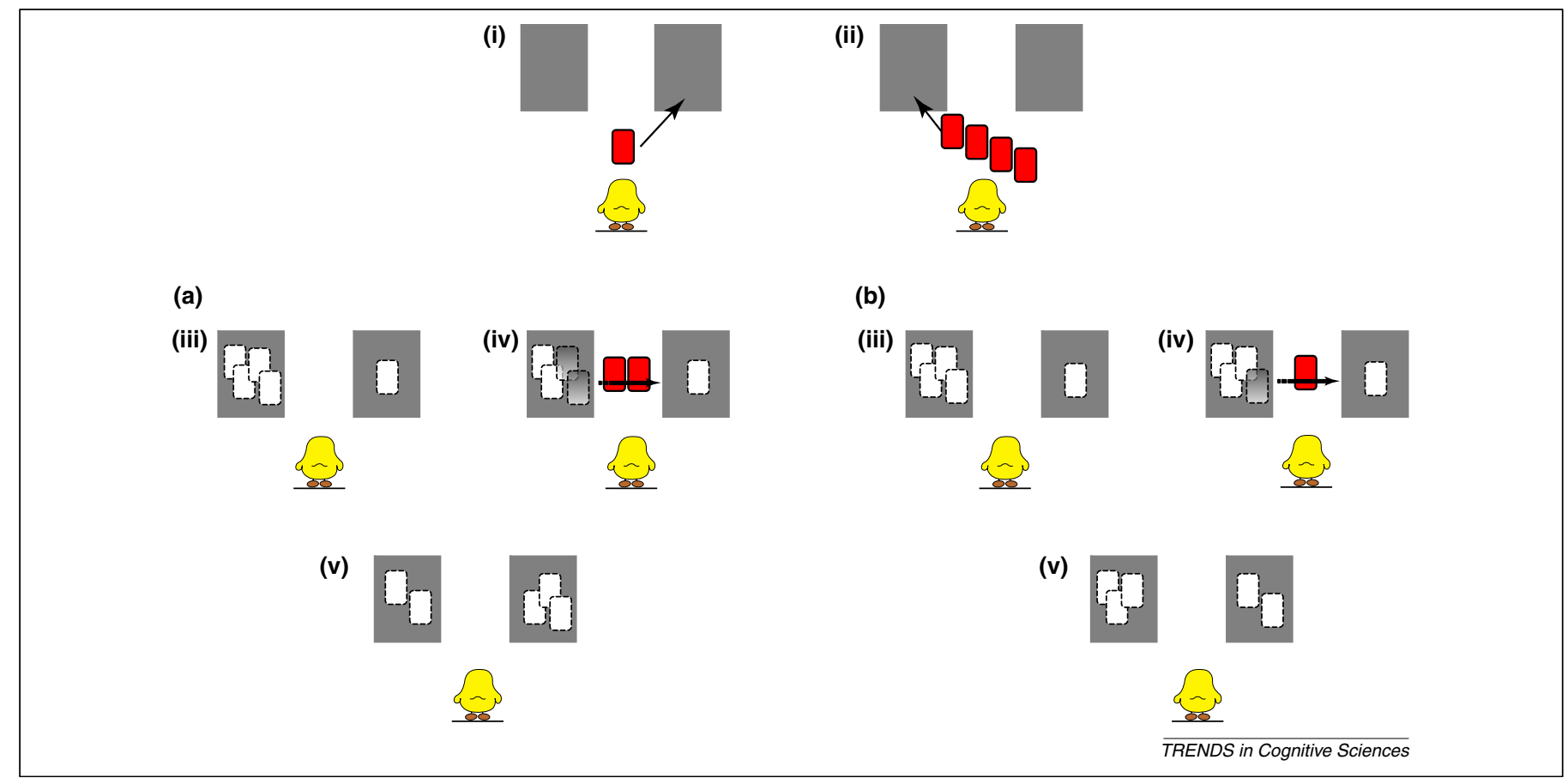

Figure 1. Arithmetic in newborn chicks. Newly hatched domestic chicks were imprinted on five identical objects and then one ball was hidden behind one screen (i) and four

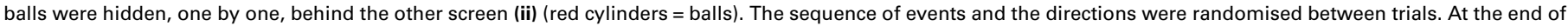
the first displacement event, therefore, either four or one ball(s) were hidden behind each screen (iii). At this point, in condition (a) two balls moved, one by one, from the screen hiding four to the one hiding a single ball (iv). At test (v), chicks approached the larger number of imprinting balls, even though the larger number of balls was not behind the screen where the larger number of balls had initially disappeared. In condition (b), only one ball moved from the screen hiding four to the one hiding a single ball (iv). At test (v) chicks rejoined the larger number of imprinting balls, which was not behind the screen where the final hiding of balls had been observed [39]. 


\section{Box 1. Phylogenetic comparative methods}

\section{Controlling for evolutionary relatedness}

Analysing diversity in cognitive ability across species requires methods that control for the hierarchical relatedness of organisms through the branching process of descent [88]. Standard statistical tests of non-independent species data will overestimate the degrees of freedom available and increase the risk of Type I error. Evolutionary biologists have therefore developed a range of computational methods to: (i) build trees (phylogenies) that describe species relationships; and (ii) track the evolution of traits on those phylogenies $[89,90]$ [phylogenetic comparative methods (PCMs)]. Trees are usually inferred from gene sequence data, but morphological [91] and behavioural [92] data can also be used. Given a phylogenetic hypothesis about historical relatedness and the variable distribution of a trait at the tree 'tips', one can use statistical approaches to infer the nature and likelihood of the underlying evolutionary processes.

\section{Reconstructing ancestral states}

The present can reveal the past: PCMs can be used to reconstruct the ancestral state of a trait (behavioural, cognitive, morphological, even cultural) for the nodes (common ancestors) in a phylogeny that describes the history of a group of species. This 'virtual archaeology' process enables researchers to establish the directionality of trait change, to test models of evolution and to incorporate independent information, such as fossil data, in hypothesis testing. Methods use the data at the tips of the trees, a tree or set of trees, and some optimality approach or model of evolution. Different methods offer a range of approaches, from basic to highly sophisticated, and are implemented in a range of software packages [93].

\section{Other questions and applications}

Both practically and principally, many evolutionary questions can only be addressed using a phylogenetic framework [94,95]: for example, the inference of ancestral states, calculating rates of evolution, assessing the degree of phylogenetic signal in the data and examining the mode of evolutionary change (e.g. punctual versus gradual). Standard regression models can be used to analyse adaptive change and correlated evolution but only after similarity owing to shared ancestry is accounted for. For comparative psychology, these methods offer great potential, as they can also be used to study intra-species variation. Within humans, ethnolinguistic groups are population entities for cultural and linguistic evolution $[96,97]$. Phylogenetic methods have also been used to study chimpanzee cultural diversity [98].

common ancestor (LCA) through descent with modification of a set of reliably reoccurring developmental resources [42]. In evolutionary biology, cross-species comparisons and historical reconstruction use a set of statistical techniques called phylogenetic comparative methods (PCM). Among other possibilities (Box 1) these methods enable researchers to reconstruct probable ancestral states of shared, but variable, cognitive traits [46-50] (Figure 2).

The power of phylogenetic inference depends on sample size (the number of species) and the completeness of the tested family of species. For humans, a complete set of species with a single common ancestor that in turn is not ancestral to any other species is the great ape clade: orangutans (Pongo pygmaeus), gorillas (Gorilla gorilla), bonobos (Pan paniscus), chimpanzees (Pan troglodytes) and humans (Homo sapiens) (Figure 3). Widespread samples of distantly related species, as they are often used in comparative analysis [47], are not always desirable. For example, including just one of the 15 lesser apes (Gibbon species [51]) will increase the sample by 1 but disproportionately violate completeness requirements (5/5 great

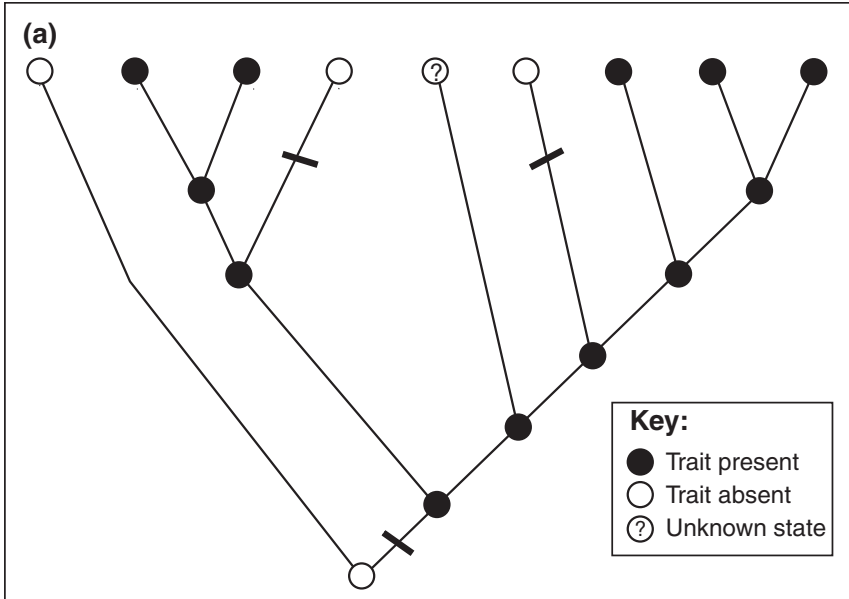

(b)

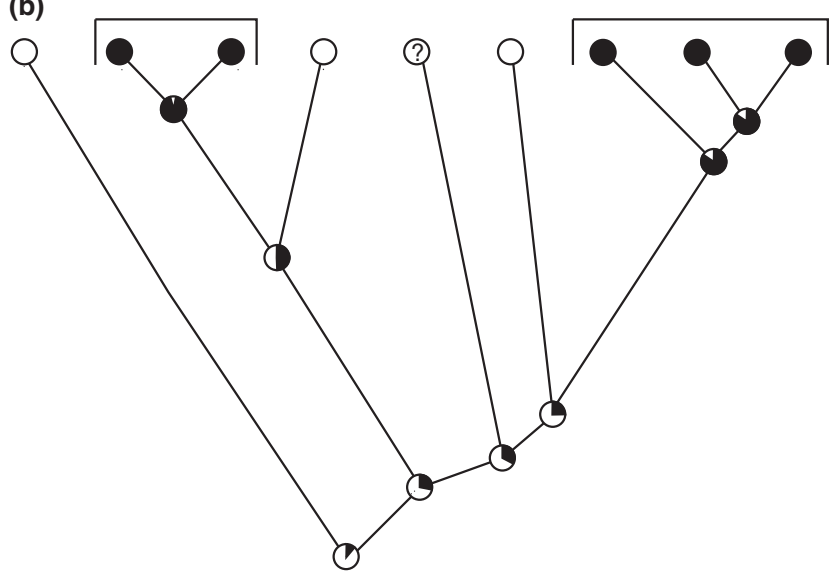

(c)

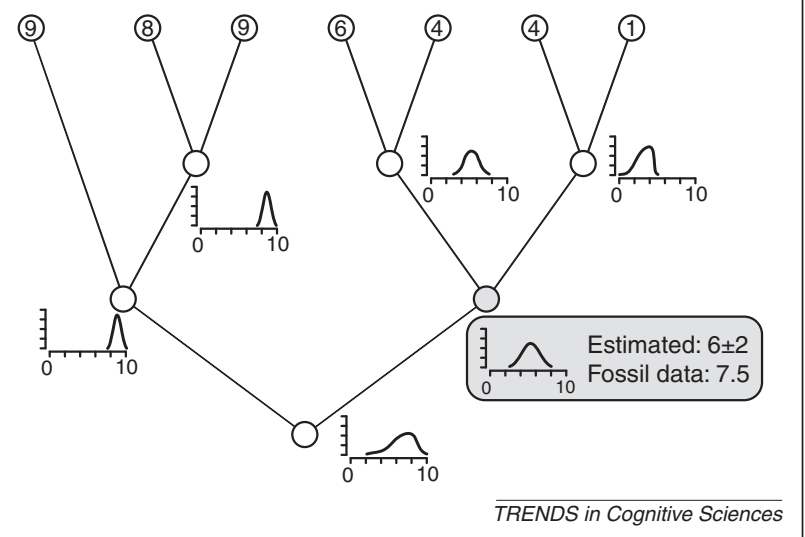

Figure 2. Inferring ancestral states by combing species data with a phylogenetic tree. The particular PCM that is used makes a difference to inferences about convergent and homologous evolution $(\mathbf{a}, \mathbf{b})$. The same set of data and species relationships are shown. Black dots represent the presence of a cognitive ability, whereas white represents absence and ? is unknown. (a) An intuitive 'eyeballing' approach similar to parsimony reconstruction minimises the number of evolutionary changes [47]. The trait is gained once and lost twice (changes = black bars), and the species can be inferred to share the trait as a result of descent (homology). Branch lengths are arbitrary. (b) The same data and phylogeny, this time using a likelihood model in which rates of gain and loss are different and change is proportional to branch length. Ancestral nodes show different reconstructions (and uncertainty) compared with those in (a). In this case, the trait might be a result of convergent evolution in the two bracketed groups. (c) Continuous data reconstructions for a morphological trait such as limb length, using a likelihood model. A large number of equally probable solutions are summarised by the distributions. Narrow curves represent certainty, whereas flatter curves show that there is ambiguity. The grey node is compared to fossil evidence; the fossil falls within the reconstruction distribution. 


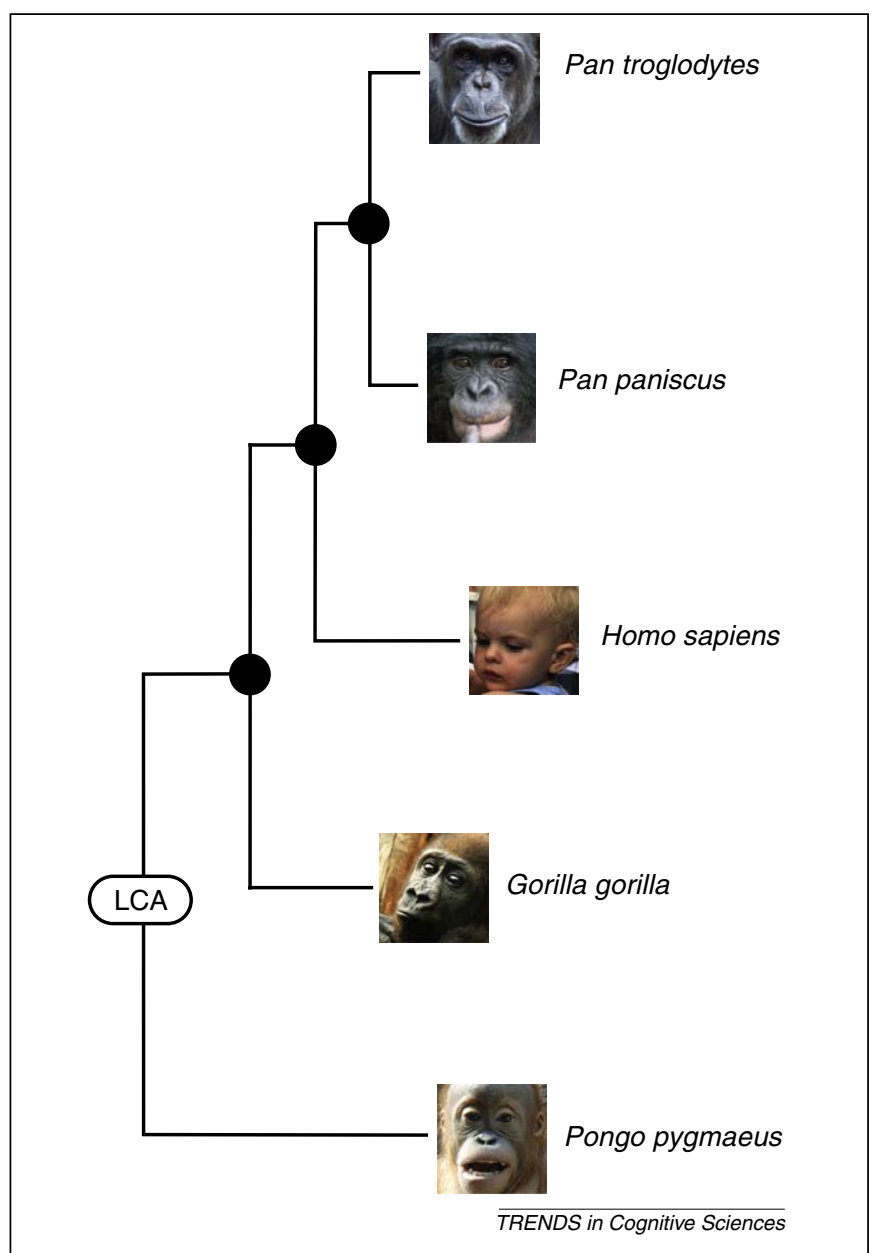

Figure 3. Consensus phylogeny of the great apes based on results from the 10 Tree Project [100]. Branch lengths are proportional to the amount of genetic change.

apes versus 6/20 apes). Sample validity is also important. Given that testing many highly endangered species is a major investment in resources, time and effort, compromises are necessary. Recent studies have attempted to increase sample validity by testing fewer species but increasing the number of individuals [52] or by sampling small numbers from multiple populations [48]. Here, we review studies that use matched methods for comparing cognition across all great apes (for reviews of cognition of space, time and number in individual primate species see [53]).

A recent study showed that all five great ape species share the ability to track the invisible displacement of hidden objects in space, but at varying levels of proficiency [54]. This kind of variation enables researchers to apply PCMs to infer the performance levels of the LCA of all great apes: the ability to track invisible displacements above chance level appears to be part of the evolutionary inheritance in all extant great apes. All great apes were also highly successful at tracking object displacements during visible rotations of a surface platform. When the rotation was invisible, that is, participants had to rely on feature cues of either the cups or the surface to infer the hidden movement, only human children above five years of age, but not younger children and no other great apes, succeeded [55]. Thus, although object tracking during rotation is a shared great ape ability, the ability to infer invisible rotations based on feature cues of either objects or the supporting surface appears to be, at least among great apes, particularly pronounced in humans.

Another cognitive domain in which humans have been claimed to be especially skilled is the domain of spatial relational reasoning $[43,56]$. All five great ape species are highly skilled when judging relations based on simple spatial rules, such as alignment and proximity. However, only children above four years of age, bonobos and chimpanzees display some mastery of reasoning by more abstract spatial-relational similarity, such as two objects being the right-most object in their respective array [48]. Mapped against their phylogenetic relationships, great ape skills in the proximity-reasoning task appear to change gradually through evolutionary history, but there appears to be a greater increase in the preference for abstract relations between the LCA of gorillas, chimpanzees and humans and the LCA of chimpanzees and humans only than in other branches on the tree. Given more data across a greater range of species, it should be possible to determine statistically where there are unusual 'punctuational' events in the evolution of cognitive capacities and preferences [57] (Figure 2a).

Tests that compare cognitive abilities across several species might suffer from the problem of unfair comparisons. Differences in ability could be dismissed by claims that experiments are simply not well adapted to suit all species equally [58]. These problems can in part be alleviated by carefully designed studies that assess performance in a test condition relative to an established control condition that all species have passed [59]. In addition, researchers can compare relative performance in the preferences among multiple solutions to the same task across species [49]. For example, all but one species (bonobos were indifferent) demonstrated a clear preference for a place-based over a feature-based memory strategy [49] in an object displacement task. Based on a phylogenetic interpretation, one can infer a preference for space over feature cues in the LCA. Note however that 'inherited' does not imply 'inflexible': great apes are, irrespective of their shared preference, able to apply feature cues successfully under different task constraints [60]. Furthermore, the preference is probably reversed in human children between one and three years of age [49]. Similarly, all great apes displayed common preferences when processing spatial relations. All great ape species, including four-year-old human children, displayed a preference for processing spatial relations using allocentric environmental cues over view-dependent egocentric cues [50].

Similar to the preference for place over feature, this preference for allocentric processing can then be inferred as part of human heritage as great apes. Inherited does not imply invariant, however: this allocentric preference not only changes across ontogeny, but also depends on the cultural context in which children grow up [50].

A similar phylogenetic perspective can be taken for the domain of number cognition. Basic performance characteristics in quantity discrimination tasks are shared across animal taxa [61], including great apes [52,62]. All tested great apes can select the larger of two quantities by 
approximation, both when presented simultanously and in sequence, even when the quantities are large and the numerical distance between them is small [62]. Similar performance levels have been reported for human children from roughly 6 years of age onwards [63], indicating a common heritage of the proximate number system [64]. Other numerical skills, such as the ability to order sets of quantities (ordinal skills) [65], might evolve in tandem with quantity discrimination (cardinal skills), or they might have independent evolutionary histories: applying PCMs to a carefully selected array of species would provide insight into the interdependence of these cognitive features (Box 1). Similar questions can be asked in the cognitive domain of time. Although little great ape research exists, it has been shown that whereas monkeys (rhesus macaques) failed to remember the 'when' component of an event, chimpanzees, bonobos (and arguably, orangutans) remembered when an event occurred [66]. Further tests with gorillas and orangutans are needed to confirm these results and thus enable investigation of the evolutionary history of time-related cognitive abilities.

\section{Which cognitive abilities are uniquely human?}

Not all cognitive traits that are shared between species are the outcome of common evolutionary history; similarities in cognitive abilities and biases can emerge independently in distantly related species (Figure 2b). These cases of convergent evolution place human cognitive skills in their evolutionary context within the animal kingdom: distantly related animal models can be used to show researchers whether complex cognitive abilities arose only once, thus producing outcomes that are shared only by descendants of a common ancestor (homologous traits), or whether these outcomes emerge independently through convergent evolution in distantly related taxa. Cases of convergent evolution also enable researchers to identify similar evolutionary pressures, thus enabling the discovery of the proximate mechanisms that produce complex equifinal outcomes in two or more lineages [67]. PCMs can arbitrate whether convergent or homologous evolution is more likely for particular traits (Figure 2b), and coevolutionary methods can test hypotheses about the relevant selective pressures acting on cognitive evolution [68].

\section{Distantly related animal models}

Mental time travel enables an individual to travel back in the mind's eye to recall previous events (episodic memory) and to travel forwards in the mind's eye to imagine future needs (episodic prospection). Many have assumed that this ability is unique to humans [45,69], particularly when episodic memory and future planning are defined in terms of the conscious experience of recollecting past events and imagining or pre-experiencing future events.

However, this is a controversial topic (most recently $[70,71])$. The absence of any agreed behavioural markers of conscious experience [72] presents an insurmountable barrier to demonstrating such cognitive skills in animal models; for how could one ever know if a nonhuman animal has a sense of self that it can project to another time [70,71]? Over the past 12 years, however, a suite of studies on birds and mammals, challenges the assumption that mental time travel is unique to humans by focusing on strictly observable behavioural criteria. Tulving's original definition of episodic memory in non-human animals identified episodic recall as the retrieval of information about three things: where a unique event took place; what occurred during the episode; and when the episode happened [73]. The advantage of this definition is that the simultaneous retrieval and integration of such tripartite information can be demonstrated behaviourally in animals. Later, the term 'episodic-like memory' was coined to refer to this ability [74].Although at least some great ape species could be shown to pass tests of what-when-where memory [66], other primates that are more distantly related to humans failed to remember the 'when' component of past episodes [75]. This pattern might be taken to indicate a recent change in homologous evolution within the primate family. However, the finding that some species of food-caching birds pass the same criteria additionally suggest an interesting case of convergent evolutionary history $[74,76]$.

There are good functional reasons for believing that food-caching birds would need to rely on specific past experiences about what happened where and when. Food-caching birds hide perishable caches as well as non-perishable ones so there would be much selective advantage in them remembering what they had cached, where, and when. A series of controlled experiments demonstrated that western scrub-jays (Aphelocoma californica) do remember what types of food caches they hid, in which spatial locations, and how long ago [74]. Moreover, the birds form integrated memories about 'what happened, where and when' rather than encoding each of these three pieces of information separately [77].

Other researchers have argued about this definition, however. Eacott and colleagues, for example, have proposed that the 'when' component simply serves as an occasion setter to identify episodic memories that occurred in different contexts, of which time is only one. Consequently, they argued that a better criterion for epsiodic-like memory is 'what-where-which' rather than 'what-where-when' because the 'when' component is only one of several possible contexts or occasion setters [78]. Others, such as Zentall and colleagues [79], argued that epsiodic recall happens automatically. In other words, at the time of encoding the information in an episodic memory, the subject does not normally know what information will need to be recalled at a later date. Zentall and colleagues give the example of what you ate for breakfast this morning. If you expect to be asked the question, you can encode an answer when you eat breakfast; and therefore when the expected question is asked, you only need to remember the prepared answer as opposed to having to recall the event itself. If the question is unexpected, you must cast your mind back to breakfast time to recall episodically the necessary information [79].

Similar to the case of episodic memory, it is possible to use behavioural criteria for the existence of forethought, but exactly what constitutes evidence for future planning is much debated. It is generally agreed that mental time travel into the future must be distinguished from other prospectively oriented but non-cognitive behaviours (such as those triggered by a seasonal cue). Three criteria are important: first, the behaviour must be shown to be 
sensitive to consequences and the animals can therefore learn to adjust their responses appropriately, for example avoiding to cache in sites that are known to be subject to pilferage. Second, the behaviours must be oriented towards a future goal, independent of current goals. Finally, the behaviour should involve true forethought, as opposed to instrumental conditioning in which the anticipatory act has previously been rewarded.

Although some primates [80,81] and corvids [82] take actions in the present based on their future consequences, these studies have not demonstrated reference to future motivational states independent of current ones [76], or without extensive reinforcement of the anticipatory act [83]. Here too, studies of western scrub-jays have provided the key empirical work, capitalising on the fact that food caching is prospective (the only benefit of caching now is to eat the food in the future). When given a novel opportunity to cache, the birds preferentially cached food in a room in which they were not given that food for breakfast relative to a food that they had received for breakfast in that room, when given these foods the evening before. The behaviour is both a novel action (i.e. that no associative learning can have occurred) and is appropriate to a motivational state other than the one the animal is in at that moment. This then meets the requirements for future planning. One might argue that the jays simply cache according to a general heuristic to balance food sources, but this does not exclude the possibility that the cognitive processes that enable them to implement this heuristic involve some form of foresight [84].

Furthermore, studies have shown that when given two foods, A and B, the birds would cache more of food A relative to food $B$ even if they are satiated on food A at the time of caching, once they have learned that when they get an opportunity to recover their caches they will be satiated on food B. This suggests that their caching decisions are motivated by what the birds want to eat at recovery rather than at caching [76]. These studies suggest that scrub-jays have the ability to take actions for the future, although it is far from clear whether they do so by mental time travel into the future.

Nonetheless, these studies suggest that some animals have the ability to take specific actions for the future. Recent work on non-human apes is substantiating this claim by showing that they can also take actions for future motivational needs $[85,86]$. At issue is whether these

\section{Box 2. Questions for future research}

- Across vertebrate species, what specific aspects of knowledge of number, space and time are available at birth in the absence of specific experiences? What neural mechanisms are responsible for their operation?

- What are the evolutionary constraints on cognition from a biological point of view? To what extent do differences in neuroarchitecture impact upon the apparent functional similarities in behaviour across distantly related species, such as apes and crows?

- How can PCMs be used to identify sets of species to maximize the power of comparisons across small sets of species [99]?

- What experimental paradigms are appropriate for comparisons across a wider range of taxa?

- Do specific cognitive abilities arise in different ecological or social contexts and can PCMs be used for coevolutionary modelling? abilities are widely spread among the animal kingdom, or whether they are exclusive to corvids and apes, and thus a product of a rare convergent evolution and, if so, what are the selective processes that were common to both corvids and apes and yet exclusive to them. More comparative studies across a greater range of species will be required to answer these types of question (Box 2). Further work might also untangle the similarities and differences in the proximate mechanisms, given such intriguing similarities in cognition, yet divergence in the brain architecture. The bird brain has a different structure to that of humans and all other mammals, bereft as it is of the six-layered structure of the human neocortex, which has long been thought to provide the unique machinery for cognition [87].

\section{Concluding remarks}

The careful selection of animal models provides exciting, novel perspectives on the development and evolution of human cognitive structure. We have reviewed here evidence from spatial, temporal and numerical cognition, all three of which are foundational cognitive domains ensuring basic vertebrate experience. In these domains, precocial animals can demonstrate how functional and complex cognition can be in place at birth without further specific experiential input. Taxonomically informed comparisons across related species enable one to identify the role of phylogeny in cognitive abilities and preferences. Finally, distantly related animal models often challenge what one might think are traits unique to humans. Cases of convergent evolution invite researchers to identify equivalent evolutionary pressures, thus enabling the discovery of the proximate mechanisms that produce complex equifinal outcomes in two or more lineages. Cross-species comparative research therefore enables cognitive science to go beyond the standard investigative toolbox and answer salient questions about the origins of the human mind and its capabilities.

\section{References}

1 Shettleworth, S.J. (2010) Cognition, Evolution, and Behavior, Oxford University Press

2 Tommasi, L. et al. (2009) Cognitive Biology, MIT Press

3 Spelke, E.S. and Kinzler, K.D. (2009) Innateness, learning, and rationality. Child Dev. Perspect. 3, 96-98

4 Gibson, E.J. and Walk, R.D. (1960) The 'visual cliff. Sci. Am. 202, 64 71

5 Spelke, E.S. and Kinzler, K.D. (2007) Core knowledge. Dev. Sci. 10, $89-96$

6 Vallortigara, G. et al. (2005) Visually inexperienced chicks exhibit spontaneous preference for biological motion patterns. PLoS Biol. 3 , e208

7 Mascalzoni, E. et al. (2010) Innate sensitivity for self-propelled causal agency in newly hatched chicks. Proc. Natl. Acad. Sci. U. S. A. 107 $4483-4485$

8 Vallortigara, G. (2006) The cognitive chicken: visual and spatial cognition in a non-mammalian brain. In Comparative Cognition: Experimental Explorations of Animal Intelligence (Wasserman, E.A and Zentall, T.R., eds), pp. 53-70, Oxford University Press

9 Vallortigara, G. and Regolin, L. (2006) Gravity bias in the interpretation of biological motion by inexperienced chicks. Curr. Biol. 16, R279-280

$10 \mathrm{Klin}$, A. et al. (2009) Two-year-olds with autism orient to non-social contingencies rather than biological motion. Nature 459, 257-261

11 Simion, F. et al. (2008) A predisposition for biological motion in the newborn baby. Proc. Natl. Acad. Sci. U. S. A. 105, 809-813 
12 Jarvis, E.D et al. (2005) Avian brains and a new understanding of vertebrate brain evolution. Nat. Rev. Neurosci. 6, 151-159

13 Cheng, K. and Newcombe, N.S. (2005) Is there a geometric module for spatial orientation? Squaring theory and evidence. Psychon. Bull. Rev. 12, 1-23

14 Vallortigara, G. (2009) Animals as natural geometers. In Cognitive Biology (Tommasi, L. et al., eds), pp. 83-104, MIT Press

15 Lee, S.A. and Spelke, E.S. (2010) A modular geometric mechanism for reorientation in children. Cogn. Psychol. 61, 152-176

16 Newcombe, N.S. and Ratliff, K.R. (2007) Explaining the development of spatial reorientation: Modularity-plus-language versus the emergence of adaptive combination. In The Emerging Spatial Mind (Plumert, J. and Spencer, J., eds), pp. 53-76, Oxford University Press

17 Cheng, K. (2008) Whither geometry? Troubles of the geometric module. Trends Cognit. Sci. 12, 355-361

18 Chiandetti, C. and Vallortigara, G. (2010) Experience and geometry: controlled-rearing studies with chicks. Anim. Cognit. 13, $463-470$

19 Nardini, M. et al. (2009) A viewpoint-independent process for spatial reorientation. Cognition 112, 241-248

20 Pecchia, T. and Vallortigara, G. (2010) Reorienting strategies in a rectangular array of landmarks by domestic chicks (Gallus gallus). J. Comp. Psychol. 124, 147-158

21 Wystrach, A. and Beugnon, G. (2009) Ants learn geometry and features. Curr. Biol. 19, 61-66

22 Lakusta, L. et al. (2010) Impaired geometric reorientation caused by genetic defect. Proc. Natl. Acad. Sci. U. S. A. 107, 2813-2817

23 Doeller, C.F. et al. (2008) Parallel striatal and hippocampal systems for landmarks and boundaries in spatial memory. Proc. Natl. Acad. Sci. U. S. A. 105, 5915-5920

24 Solstad, T. et al. (2008) Representation of geometric borders in the entorhinal cortex. Science 322, 1865-1868

25 Chiandetti, C. and Vallortigara, G. (2008) Is there an innate geometric module? Effects of experience with angular geometric cues on spatial re-orientation based on the shape of the environment. Anim. Cognit. $11,139-146$

26 Langston, R.F. et al. (2010) Development of the spatial representation system in the rat. Science $328,1576-1580$

27 Wills, T.J.et al. (2010) Development of the hippocampal cognitive map in preweanling rats. Science $328,1573-1576$

28 Regolin, L. and Vallortigara, G. (1995) Perception of partly occluded objects by young chicks. Percept. Psychophys. 57, 971-976

29 Kellman, P.J. and Arterberry, M.E. (1998) The Cradle of Knowledge, MIT Press

30 Valenza, E. et al. (2006) Perceptual completion in newborn human infants. Child. Dev. 77, 1810-1821

31 Vallortigara, G. et al. (2009) Doing Socrates experiment right: controlled rearing studies of geometrical knowledge in animals. Curr. Opin. Neurobiol. 19, 20-26

32 Pyers, J.E. et al. (2010) Evidence from an emerging sign language reveals that language supports spatial cognition. Proc. Natl. Acad. Sci. U. S. A. $107,12116-12120$

33 Brown, A.A. et al. (2007) Growing in circles: rearing environment alters spatial navigation in fish. Psychol. Sci. 18, 569-573

34 Feigenson, L. et al. (2002) Infants' discrimination of number vs. continuous extent. Cogn. Psychol. 44, 33-66

35 Cordes, S. and Brannon, E.M. (2009) The relative salience of discrete and continuous quantity in young infants. Dev. Sci. 12, 453-463

36 Feigenson, L. (2005) A double-dissociation in infants' representations of object arrays. Cognition 95, B37-B48

37 Rugani, R. et al. (2010) Is it only humans that count from left to right? Biol. Lett. 6, 290-292

38 Rugani, R. et al. (2008) Discrimination of small numerosities in young chicks. J. Exp. Psychol. Anim. Behav. Process. 34, 388-399

39 Rugani, R. et al. (2009) Arithmetic in newborn chicks. Proc. R. Soc. Biol. Sci. 276, 2451-2460

40 Diekamp, B. et al. (2005) A left-sided visuospatial bias in birds. Curr. Biol. 15, R372-373

41 Karmiloff-Smith, A. (1992) Beyond Modularity: A Developmental Perspective on Cognitive Science, The MIT Press

42 Mameli, M. and Bateson, P. (2006) Innateness and the sciences. Biol. Philos. 21, 155-188
43 Gentner, D. (2003) Why we're so smart. In Language in Mind: Advances in Study of Language and Thought (Gentner, D. and Goldin-Meadow, S., eds), pp. 195-235, The MIT Press

44 Wellman, H.M. et al. (2001) Meta-analysis of theory-of-mind development: the truth about false belief. Child. Dev. 72, 655-684

45 Tulving, E. (2002) Episodic memory: from mind to brain. Annu. Rev. Psychol. 53, 1-25

46 Byrne, R.W. (1995) The Thinking Ape: Evolutionary Origins of Intelligence, Oxford University Press

47 Fitch, W.T. et al. (2010) Social cognition and the evolution of language: constructing cognitive phylogenies. Neuron $65,795-814$

48 Haun, D.B.M. and Call, J. (2009) Great apes' capacities to recognize relational similarity. Cognition 110, 147-159

49 Haun, D.B.M. et al. (2006) Evolutionary psychology of spatial representations in the hominidae. Curr. Biol. 16, 1736-1740

50 Haun, D.B.M. et al. (2006) Cognitive cladistics and cultural override in hominid spatial cognition. Proc. Natl. Acad. Sci. U. S. A. 103, 1756817573

51 Groves, C.P. (2001) Primate Taxonomy, Smithsonian Institution Press

52 Herrmann, E. et al. (2007) Humans have evolved specialized skills of social cognition: the cultural intelligence hypothesis. Science 317 , $1360-1366$

53 Seed, A. and Tomasello, M. (2010) Primate cognition. Topics Cogn. Sci. 2, 407-419

54 Barth, J. and Call, J. (2006) Tracking the displacement of objects: a series of tasks with great apes (Pan troglodytes, Pan paniscus, Gorilla gorilla, and Pongo pygmaeus) and young children (Homo sapiens). J. Exp. Psychol. Anim. B 32, 239-252

55 Okamoto-Barth, S. and Call, J. (2008) Tracking and inferring spatial rotation by children and great apes. Dev. Psychol. 44, 1396-1408

56 Penn, D.C. et al. (2008) Darwin's Mistake: explaining the discontinuity between human and nonhuman minds. Behav. Brain Sci. $31,109-130$

57 Pagel, M. (1999) Inferring the historical patterns of biological evolution. Nature 401, 877-884

58 Boesch, C. (2007) What makes us human (Homo sapiens)? The challenge of cognitive cross-species comparison. J. Comp. Psychol. $121,227-240$

59 Tomasello, M. and Call, J. (2008) Assessing the validity of ape-human comparisons: a reply to Boesch (2007). J. Comp. Psychol. 122, 449-452

60 Kanngiesser, P. and Call, J. (2010) Bonobos, chimpanzees, gorillas, and orang utans use feature and spatial cues in two spatial memory tasks. Anim. Cognit. 13, 419-430

61 Cantlon, J.F. et al. (2009) Beyond the number domain. Trends Cognit. Sci. 13, 83-91

62 Hanus, D. and Call, J. (2007) Discrete quantity judgments in the great apes (Pan paniscus, Pan troglodytes, Gorilla gorilla, Pongo pygmaeus): the effect of presenting whole sets versus item-by-item. J. Comp. Psychol. 123, 227-240

63 Halberda, J. and Feigenson, L. (2008) Developmental change in the acuity of the 'Number Sense': The Approximate Number System in 3-, 4-, 5-, and 6-year-olds and adults. Dev. Psychol. 44, 1457-1465

64 Feigenson, L. et al. (2004) Core systems of number. Trends Cognit. Sci. $8,307-314$

65 Brannon, E.M. and Terrace, H.S. (1998) Ordering of the numerosities 1 to 9 by monkeys. Science $282,746-749$

66 Martin-Ordas, G. et al. (2010) Keeping track of time: evidence for episodic-like memory in great apes. Anim. Cognit. 13, 331-340

67 Seed, A. et al. (2009) Intelligence in corvids and apes: a case of convergent evolution? Ethology 115, 401-420

68 Hare, B. and Tomasello, M. (2005) Human-like social skills in dogs? Trends Cognit. Sci. 9, 439-444

69 Suddendorf, T. and Corballis, M.C. (1997) Mental time travel and the evolution of the human mind. Genet. Soc. Gen. Psychol. Monogr. 123, 133-167

70 Roberts, W.A. and Feeney, M.C. (2009) The comparative study of mental time travel. Trends Cognit. Sci. 13, 271-277

71 Suddendorf, T. and Corballis, M.C. (2010) Behavioural evidence for mental time travel in nonhuman animals. Behav. Brain Res. 215, 292 298

72 Griffiths, D. et al. (1999) Episodic memory: what can animals remember about their past? Trends Cognit. Sci. 3, 74-80 
73 Tulving, E. (1972) Episodic and semantic memory. In Organisation of Memory (Tulving, E. and Donaldson, W., eds), pp. 381-403, Academic Press

74 Clayton, N.S. and Dickinson, A. (1998) Episodic-like memory during cache recovery by scrub jays. Nature $395,272-274$

75 Hampton, R.R. et al. (2005) Rhesus monkeys (Macaca mulatta) demonstrate robust memory for what and where, but not for when, in an open-eld test of memory. Learn. Motiv. 36, 245-259

76 Correia, S.P. et al. (2007) Western scrub-jays anticipate future needs independently of their current motivational state. Curr. Biol. 17, 856861

77 Clayton, N.S. et al. (2003) Can animals recall the past and plan for the future? Nat. Rev. Neurosci. 4, 685-691

78 Eacott, M.J. and Norman, G. (2004) Integrated memory for object, place, and context in rats: a possible model of episodic-like memory? $J$. Neurosci. 24, 1948-1953

79 Zentall, T.R. et al. (2008) Episodic-like memory: pigeons can report location pecked when unexpectedly asked. Behav. Proc. 79, 93-98

80 Mulcahy, N.J. and Call, J. (2006) Apes save tools for future use. Science 312, 1038-1040

81 Naqshbandi, M. and Roberts, W.A. (2006) Anticipation of future events in squirrel monkeys (Saimiri sciureus) and rats (Rattus norvegicus): tests of the Bischof-Kohler hypothesis. J. Comp. Psychol. 120, 345-357

82 de Kort, S.R. et al. (2007) The control of food-caching behavior by western scrub-jays (Aphelocoma californica). J. Exp. Psychol. Anim. Behav. Process. 33, 361-370

83 Raby, C.R. et al. (2007) Planning for the future by western scrub-jays. Nature 445, 919-921

84 Raby, C.R. and Clayton, N.S. (2009) Prospective cognition in animals. Behav. Proc. 80, 314-324

85 Osvath, M. (2009) Spontaneous planning for future stone throwing by a male chimpanzee. Curr. Biol. 19, R190-191
86 Osvath, M. and Osvath, H. (2008) Chimpanzee (Pan troglodytes) and orangutan (Pongo abelii) forethought: self-control and pre-experience in the face of future tool use. Anim. Cognit. 11, 661-674

87 Emery, N.J. and Clayton, N.S. (2004) The mentality of crows: convergent evolution of intelligence in corvids and apes. Science 306, 1903-1907

88 Harvey, P.H. and Pagel, M.D. (1991) The Comparative Method in Evolutionary Biology, Oxford University Press

89 Felsenstein, J. (2004) Inferring Phylogenies, Sinauer Associates

90 Lemey, P. et al., eds (2009) The Phylogenetic Handbook: A Practical Approach to Phylogenetic Analysis and Hypothesis Testing, Cambridge University Press

91 Wiens, J.J. (ed.) (2000) Phylogenetic Analysis of Morphological Data, Smithsonian Institution Press

92 Kennedy, M. et al. (1996) Hop, step and gape: do the social displays of the Pelecaniformes reflect phylogeny? Anim. Behav. 51, 273-291

93 Nunn, C.L. (2011) The Comparative Approach in Evolutionary Anthropology. University of Chicago Press. (in press)

94 Capellini, I. et al. (2009) Does sleep play a role in memory consolidation? A comparative test. PLoS One 4, e4609

95 Reader, S.M. and Laland, K.N. (2002) Social intelligence, innovation, and enhanced brain size in primates. Proc. Natl. Acad. Sci. U. S. A. 99, $4436-4441$

96 Mace, R. and Holden, C.J. (2005) A phylogenetic approach to cultural evolution. Trends Ecol. Evol. 20, 116-121

97 Pagel, M. (2009) Human language as a culturally transmitted replicator. Nat. Rev. Genet. 10, 405-415

98 Lycett, S.J. et al. (2009) Cladistic analyses of behavioural variation in wild Pan troglodytes: exploring the chimpanzee culture hypothesis. $J$. Human Evol. 57, 337-349

99 Arnold, C. and Nunn, C.L. (2010) Phylogenetic targeting of research effort in evolutionary biology. Am. Nat. 176, 601-612

100 Arnold, C. et al. (2010) The 10k Trees Website: a new online resource for primate phylogeny. Evol. Anthropol. 19, 114-118 